\title{
Investigación en relaciones públicas en España
}

\section{Public relations research in Spain}

\author{
Antonio Castillo-Esparcia; Carmen Carretón-Ballester; Paula Pineda-Martínez
}

Cómo citar este artículo:

Castillo-Esparcia, Antonio; Carretón-Ballester, Carmen; Pineda-Martínez, Paula (2020). “Investigación en relaciones públicas en España". Profesional de la información, v. 29, n. 3, e290330.

https://doi.org/10.3145/epi.2020.may.30

Artículo recibido el 10-11-2019

Aceptación definitiva: 08-06-2020

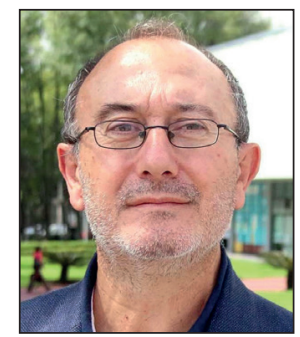

Antonio Castillo-Esparcia

https://orcid.org/0000-0002-9751-8628

Universidad de Málaga

Departamento de Comunicación

Audiovisual y Publicidad

Campus de Teatinos, $\mathrm{s} / \mathrm{n}$.

29071 Málaga, España

acastilloe@uma.es

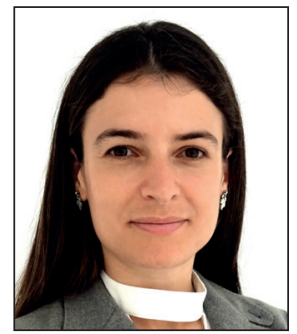

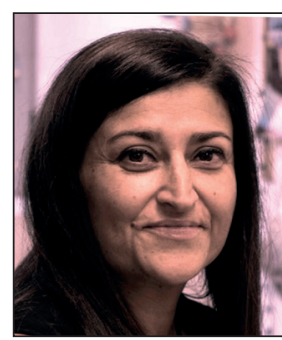

Carmen Carretón-Ballester

https://orcid.org/0000-0002-4851-9683

Universidad de Alicante, Departamento de Comunicación y Psicología Social

Ctra. de San Vicente del Raspeig, s/n. 03690 San Vicente del Raspeig (Alicante), España

mc.carreton@ua.es

\section{Resumen}

Entre 2004 y 2006 se constituyeron en España las dos asociaciones científicas más relevantes para los académicos e investigadores de relaciones públicas y de la comunicación con la misión de potenciar y difundir la investigación y promover foros y redes de intercambio científico: la Asociación de Investigadores en Relaciones Públicas (AIRP) y la Asociación Española de Investigación de la Comunicación $(A E-I C)$. Con el estudio de las tesis doctorales defendidas y de las contribuciones a los congresos internacionales que organizan ambas asociaciones en el período comprendido entre 2004 y 2018 , se persigue el objetivo principal de determinar, describir y analizar la investigación en relaciones públicas como objeto del debate científico de la disciplina en España. Para ello, se utiliza el análisis de contenido como método cualitativo y cuantitativo de las ciencias sociales. Entre los resultados más relevantes destacan el predominio de mujeres investigadoras en el campo, las relaciones públicas y comunicación digital como temática mayoritaria, el uso de una metodología mixta y el análisis de contenido como principal técnica de investigación. El diagnóstico de su evolución y de cómo se gestiona la investigación de las relaciones públicas en España define las tendencias y los retos como resultado del estudio.

\section{Palabras clave}

Relaciones públicas; Investigación; Metodología; Congresos; Investigadores; Tesis doctorales; Diagnóstico; Comunicación; Asociaciones científicas; España.

\begin{abstract}
Between 2004 and 2006, the two most relevant scientific associations for academics and researchers in the field of public relations and communication were set up in Spain with the mission of strengthening and disseminating research and promoting forums and networks for scientific exchange: the Association of Researchers in Public Relations (AIRP) and the Spanish Association for Communication Research $(A E-I C)$. Based on a study of the doctoral theses presented and contributions to international conferences organized by both associations in the period between 2004 and 2018 , our main objective is to determine, describe, and analyze research trends in the academic field of public relations in the Spanish context. To this end, content analysis is applied as a qualitative and quantitative method in the social sciences. Among the most relevant results, the following stand out: the predominance of women researchers in the field, public
\end{abstract}


relations and digital communication as the main topics, the use of mixed methodology, and content analysis as the main research technique. The diagnosis of the evolution and management of public relations research in Spain presented herein defines the corresponding trends and challenges.

\section{Keywords}

Public relations; Research; Methodology; Congresses; Researchers; Doctoral theses; Diagnosis; Communication; Scientific associations; Spain.

\section{Introducción}

El estudio de la evolución de las relaciones públicas en España no es una tarea fácil. Su desarrollo ha sido lento y costoso, entre otras cuestiones, debido a la corta edad de esta disciplina y profesión en nuestro país (Arceo-Vacas, 2006). No es hasta finales de la dictadura franquista que surgen las primeras Facultades de Ciencias de la Información, constituyéndose como principales actores de la formación y producción investigadora a través de congresos y publicaciones científicas (Castillo-Esparcia, 2011). No obstante, el interés tanto profesional, como académico e investigador de las relaciones públicas ha crecido de manera extraordinaria en el país en los últimos años.

Los congresos científicos y las tesis doctorales son algunos de los principales indicadores de esta evolución. Sin embargo, y especialmente en el caso de los congresos, su estudio ha recibido muy poca atención por parte de la academia en comparación con las publicaciones en revistas científicas, y ya en 2014 Míguez-González, Baamonde-Silva y Corbacho-Valencia (2014) realizaban un llamado a investigarlos. El presente artículo pretende describir la evolución de la investigación en relaciones públicas para mostrar cómo la teoría y la práctica han contribuido a una mejora de la comunicación como componente estratégico y directivo de las organizaciones. Así, se analizan en primera instancia las comunicaciones presentadas de 2004 a 2018 en el único congreso científico centrado de manera exclusiva en relaciones públicas en España, el "Congreso internacional de investigación en relaciones públicas" de la Asociación de Investigadores en Relaciones Públicas, así como las comunicaciones presentadas durante el mismo período de tiempo a uno de los principales congresos sobre comunicación en el país, el "Congreso internacional de la asociación española de investigadores de la comunicación (AE-IC)". En segundo orden, se examinan en el mismo rango temporal las tesis publicadas en España sobre relaciones públicas.

\section{Estudios y tendencias de investigación en relaciones públicas en España}

El interés en examinar el estado actual de las relaciones públicas ha crecido de manera exponencial en los últimos años. A nivel internacional, encontramos una gran parte de estudios que se han centrado en analizar la investigación en relaciones públicas de manera genérica, examinando aspectos como la autoría, los temas, la metodología y los marcos teóricos de las investigaciones (Molleda; Laskin, 2005; Meadows; Meadows, 2014; Míguez-González; Corbacho-Valencia; Baamonde-Silva, 2016; Morkunas; Moore; Duncan, 2018). Otra parte de estos estudios se ha dedicado a analizar cuestiones más específicas, como internet y los nuevos medios (Duhé, 2012; 2015; Ye; Ki, 2012; McCorkindale; DiStaso, 2014; Wang, 2015), comunicación de crisis (Kim; Avery; Lariscy, 2009; Ha; Riffe, 2015; Liu; Bartz; Duke, 2016), asuntos públicos y lobby (Davidson, 2015), diplomacia pública (Vanc; Fitzpatrick, 2016), relaciones públicas sin ánimo de lucro (Sisco; Pressgrove; Collins, 2013), gestión estratégica de las relaciones públicas (Kim; Ni, 2013; Compte-Pujol; Matilla; Hernández, 2018), el concepto de "diálogo" o la comunicación dialógica en las relaciones públicas (Theunissen; Noordin, 2012; Morehouse; Saffer, 2018), la teoría dialógica de las relaciones públicas (McAllister-Spooner, 2009), la relación organización-públicos (Ki; Shin, 2006), o la conexión entre la inteligencia estratégica y las relaciones públicas (Santa-Soriano; Lorenzo-Álvarez; Torres-Valdés, 2018).

En el caso de España, la mayoría de los estudios sobre el estado de la investigación en relaciones públicas podrían ser ubicados en el primer grupo de investigaciones ya que, sobre todo, se han preocupado por analizar cuestiones generales de las investigaciones (autoría, temas, metodología y marcos teóricos), tanto en artículos (Míguez-González, 2011; Míguez-González, Baamonde-Silva; Corbacho-Valencia, 2014; Míguez-González; Corbacho-Valencia; Baamonde-Silva, 2016; Míguez-González; Costa-Sánchez, 2019), como en tesis doctorales (Xifra; Castillo-Esparcia, 2006; Cuenca-Fontbona; Matilla; Compte-Pujol, 2019; Miquel-Segarra; Gonçalves, 2019) y en programas de doctorado (Cuenca-Fontbona; Matilla; Compte-Pujol, 2019). No se han encontrado, sin embargo, estudios que aborden la producción de investigación en el caso de los congresos científicos. Aunque no centrados específicamente en las relaciones públicas, también encontramos otro conjunto considerable de estudios que analizan la comunicación en general (Castillo-Esparcia; Carretón-BaIlester, 2010; Masip, 2011; Fernández-Quijada, 2011a, 2011b; Castillo-Esparcia; Rubio-Moraga; Almansa-Martínez, 2012; Martínez-Nicolás; Saperas-Lapiedra, 2011; 2016; Martínez-Nicolás; Saperas-Lapiedra; Carrasco-Campos, 2019).

Una de las cuestiones fundamentales a tener en cuenta al hablar de investigación en relaciones públicas en el caso de España es que hasta los años 80 no es posible hablar sobre la práctica de esta disciplina desde un enfoque científico. Este hecho trajo consigo que el desarrollo de la investigación teórica y aplicada también fuera tardío y limitado (Arceo-Vacas, 2006). A juzgar por las investigaciones realizadas hasta el momento, la investigación académica de las relaciones públicas parece presentar ciertas carencias que no evolucionan en gran medida a lo largo del tiempo. 
Míguez-González (2011) encontró que las relaciones públicas son un tema secundario para las principales revistas de comunicación en España, con un porcentaje muy pequeño de artículos centrados total o parcialmente en esta área (5,24\%). Tres años más tarde, Míguez-González, Baamonde-Silva y Corbacho-Valencia (2014) recogieron el testigo y confirmaron su conclusión analizando las revistas de medios y comunicación más importantes en el país. Esta tendencia también se cumple en el caso de las tesis doctorales, si bien se constata un crecimiento constante a lo largo de los años. Así, en el período comprendido entre 1965 y 2005 Castillo-Esparcia y Xifra (2006) identificaron 38 tesis con el descriptor "relaciones públicas", mientras que de 2006 a 2017 la cifra ascendió a 65 tesis (Cuenca-Fontbona; Matilla; Compte-Pujol, 2019). Esta cifra es especialmente positiva, teniendo en cuenta, además, la poca oferta de programas de doctorado existentes en el país especializados en relaciones públicas (únicamente 4) (Cuenca-Fontbona; Matilla; Compte-Pujol, 2019).

Otro hallazgo de especial interés en varios de estos estudios es la invisibilidad del término "relaciones públicas" en los artículos sobre esta disciplina, que no parece haber evolucionado a lo largo del tiempo (Míguez-González, 2011; Míguez-González; Baamonde-Silva; Corbacho-Valencia, 2014). En el caso de las tesis doctorales, se ha detectado que, aunque el término "relaciones públicas" es el más utilizado, otros como "comunicación corporativa" comienzan a consolidarse en los últimos años (Miquel-Segarra; Gonçalves, 2019).

La diversidad de autores, las universidades de procedencia y los temas en las investigaciones sobre relaciones públicas también han sido confirmadas en el caso de los artículos de investigación (Míguez-González; Baamonde-Silva; Corbacho-Valencia, 2014). Sin embargo, en lo que se refiere a las tesis doctorales, se ha encontrado que una gran parte de las mismas ha sido defendida en la Universidad de Málaga (Cuenca-Fontbona; Matilla; Compte-Pujol, 2019; Miquel-Segarra; Gonçalves, 2019), relegando a la Universidad Complutense, que en anteriores estudios se encontraba primera en el ranking (Xifra; Castillo-Esparcia, 2006), a un segundo puesto. También se ha advertido que en este tipo de trabajos la mayoría de los temas de investigación se refieren a la dimensión técnica e instrumental de las relaciones públicas, dejando a un lado la parte estratégica (Xifra; Castillo-Esparcia, 2006; Cuenca-Fontbona; Matilla; Compte-Pujol, 2019; Miquel-Segarra; Gonçalves, 2019). Este hecho parece estar en línea con los actuales planes de estudio de los grados en Publicidad y relaciones públicas, que favorecen las asignaturas publicitarias en detrimento de las de relaciones públicas (Xifra; Castillo-Esparcia, 2006).

\section{Las relaciones públicas en la educación superior en España}

Después de la creación de las facultades de Ciencias de la Información en 1971, tres años más tarde, el Gobierno de España aprueba provisionalmente el Plan de estudios de la Licenciatura de Publicidad y relaciones públicas, si bien no vería su consolidación hasta 1991 (Castillo-Esparcia; Xifra, 2006).

A partir de 1992, cuando se establecen las titulaciones de las licenciaturas en Periodismo, Comunicación Audiovisual y Publicidad y relaciones públicas en las facultades de Ciencias de la Información de España, muchos planes de estudio de las dos últimas licenciaturas ya contaban con asignaturas relacionadas con las relaciones públicas y la comunicación organizacional (Noguero-Grau, 2004). Sin embargo, la obligatoriedad de convivencia en una misma licenciatura de dos campos diferentes dio lugar a un desequilibrio en detrimento de las relaciones públicas (Castillo-Esparcia; Xifra, 2006).

En 2006, previamente a la implantación del Espacio Europeo de Educación Superior (EEES), los estudios de relaciones públicas eran ofertados en 32 universidades españolas, y las asignaturas específicas de esta materia no excedían de dos, tres o cuatro (Huertas; Fernández-Cavia, 2006). Además, de los 195 académicos pertenecientes al área de conocimiento de Comunicación Audiovisual y Publicidad, tan sólo 38 eran investigadores y académicos de relaciones públicas (Xifra, 2007).

Tras examinar la presencia de las relaciones públicas en los planes de estudio previos a la implantación del Proceso de Bolonia, Xifra ya concluía que

"Las relaciones públicas son el tema invisible en las universidades españolas" (Xifra, 2007, p. 212),

y establecía que estas titulaciones resultaban insuficientes a la hora de proporcionar una educación fuerte al estudiantado en este ámbito.

Con la implantación del EEES, la oferta formativa de relaciones públicas pasó a estar presente en los tres niveles de formación superior (grados, postgrados y programas de doctorado). Aprovechando esta situación, la comunidad académica y científica en esta área realizó sendos esfuerzos, entre otros, a través de la Asociación de Investigadores en Relaciones Públicas $(A I R P)$, asesorando sobre la implementación de nuevos grados, realizando un seguimiento de la misma, y estableciendo contacto con miembros del gobierno y sector profesional (Xifra, 2007).

Sin embargo, tras la implantación del EEES, en los nuevos grados en Publicidad y relaciones públicas del curso 2009/2010 tan sólo el 19,12\% de las asignaturas eran específicas de este campo (Matilla; Hernández; Losada, 2010). Esta falta de cambios notables, tanto con el correspondiente mantenimiento de la denominación de la titulación, como con la subsis- 
tencia de la subordinación de los contenidos de relaciones públicas a los de la publicidad y su carácter fundamentalmente instrumental (no estratégico), ha sido señala de manera consecutiva por diversos autores (Matilla; Hernández, 2011; Hellín-Ortuño; García-López, 2011; Matilla; Hernández, 2012; Moreno-Fernández; Carrasco-Campos; Saperas-Lapiedra, 2014).

Más recientemente, en los planes de estudio de los grados del curso 2017/2018, se identificaron 33 grados con los términos "publicidad" y "relaciones públicas" en su denominación, y un 31,01\% de asignaturas específicas de relaciones públicas frente a un 68,99\% de publicidad (Matilla; Hernández; Compte-Pujol, 2018).

También es necesario tener en cuenta la presencia de formación específica de postgrado en relaciones públicas, que determinan la deseable perspectiva gerencial de las relaciones públicas. En este sentido, en el curso 2008/2009, tan sólo se registraron cuatro títulos oficiales, perteneciendo dos de los mismos a la misma universidad y únicamente uno de una universidad pública. Sin embargo, la oferta de títulos universitarios propios fue significativamente mayor (20 títulos), aunque por debajo del resto de títulos en el área de comunicación. Además, sólo dos de los títulos incluían "relaciones públicas" en su denominación (Hernández; Losada; Matilla, 2009).

En el curso 2016/2017 se encontró que ningún máster universitario español tipo MBA contemplaba las relaciones públicas ni comunicación corporativa como un área funcional independiente. Además, se concluyó que los programas formativos no incluían suficientes materias para la adquisición de unos conocimientos, competencias, capacidades y habilidades adecuados (Matilla; Cuenca-Fontbona; Compte-Pujol, 2018).

En suma, en base a la bibliografía revisada, aunque la necesidad de profesionales de relaciones públicas cualificados es mayor que nunca y su consejo a la alta dirección de las organizaciones sigue siendo fundamental (Armendáriz, 2015), esta actividad continúa contando con algunos problemas. Estos incluyen, entre otros:

- el hecho de que gran parte de la educación y la práctica en relaciones públicas continúa enfocándose más en la parte técnica o instrumental que en la estratégica;

- el campo continúa siendo denominado por una variedad de nombres diferentes (raramente relaciones públicas);

- la mayoría de quienes trabajan en las relaciones públicas no han sido educados específicamente en esta área (Dircom, 2018);

- los grados de Publicidad y relaciones públicas continúan dando más peso a la primera disciplina en sus programas; y, finalmente,

- el alumnado que recibe esta formación no termina plenamente cualificado o sale con importantes carencias (Perlado-Lamo-de-Espinosa; Saavedra-Llamas, 2017).

\section{Metodología}

\subsection{Objetivos del objeto de análisis}

El objetivo principal del trabajo es determinar, describir y analizar la investigación en relaciones públicas como objeto del debate científico de la disciplina en España. Para ello, se plantean los siguientes objetivos específicos:

1. Analizar la presencia de la disciplina de las relaciones públicas dentro del ámbito de la comunicación, así como las temáticas objeto de estudio de los investigadores.

2. Representar la tipología de los investigadores en relaciones públicas.

3. Mostrar el grado de empirismo científico de las aportaciones, así como describir la metodología de investigación utilizada en materia de relaciones públicas.

4. Averiguar las posibles relaciones entre las variables que analizan las tendencias investigadoras.

\subsection{Muestra}

Las unidades de registro de muestreo intencional son los repositorios de la $A I R P$ y de la $A E-I C$, a los que se ha accedido a través de los sitios web oficiales de las asociaciones y, en el caso de las tesis doctorales, la base de datos Teseo.

http://airrpp.org

https://ae-ic.org

https://www.educacion.gob.es/teseo

El congreso de la AIRP, asociación fundada en 2004, es el único congreso internacional en España centrado de manera exclusiva en la investigación en relaciones públicas. El congreso de la $A E-I C$, asociación creada en el año 2006, resulta de especial interés por ser el principal congreso sobre investigación en comunicación en el país y cuenta, además, con una sección específica sobre comunicación estratégica y organizacional.

Al seleccionar todos los trabajos del ámbito de estudio de las relaciones públicas, desde el 2004 y hasta el 2018 , la muestra coincide con el universo objeto de estudio. Se analizan 640 trabajos sobre relaciones públicas presentados en estas dos unidades de registro intencionales. El análisis revela que el $61,4 \%$ corresponde a la $A I R P$ y el $38,6 \%$ a la $A E-I C$.

Las comunicaciones que abordan las relaciones públicas de manera tangencial y no pueden ubicarse de manera clara dentro de este ámbito de estudio no fueron incluidas en el análisis. 
Para el análisis de las tesis doctorales se ha consultado la base de datos Teseo perteneciente al Ministerio de Educación, ya que recoge todas las tesis presentadas en las universidades españolas. El período analizado abarca desde 2005 a 2018, recurriendo a términos de búsqueda de relaciones públicas en los descriptores de las tesis doctorales.

\subsection{Método}

Para la recogida de información objetiva y sistemática, el método utilizado ha sido el análisis de contenido

"como técnica que combina intrínsecamente, y de ahí su complejidad, la observación y producción de los datos, y la interpretación o análisis de los datos" (Andréu-Abela, 2000, p. 2).

Las unidades de contexto del análisis de contenido para los congresos residen en los trabajos sobre relaciones de las organizaciones con sus públicos atendiendo a la bibliografía objeto de estudio y, a tal fin, se analizan los que se refieren a: relaciones públicas; relaciones; públicos; comunicación corporativa; comunicación institucional; comunicación organizacional/empresarial; y comunicación en general.

La matriz de análisis cuenta con 15 categorías nominales que persiguen clasificar los trabajos (formato; palabra clave título; palabras clave1; palabras clave2; año; autores; metodología; método cuestionario; método entrevista profundidad; método grupo de discusión; método análisis de contenido; método análisis de discurso; método focus group; método observación; método experimento; método estudio de caso), y 4 categorías ordinales (género autor1; género autor2; género autor3; universidad autor1; universidadautor2; universidad autor3; país autor1; país autor2; país autor3; temática1; tematica2) que pretenden, además de clasificarlos, ordenarlos siguiendo un orden establecido. Dados los objetivos analíticos y descriptivos de la investigación, su aplicación es cuantitativa y cualitativa. El tratamiento de los datos se analiza e interpreta con el programa estadístico SPSS v.22.

En la base de datos Teseo se analizan 103 tesis doctorales en una matriz cuyas unidades de análisis son: género, fecha de publicación, universidad, temática de estudio, metodología, idioma y mención internacional.

\section{Resultados}

\subsection{Relaciones públicas en los congresos científicos}

\subsubsection{Palabras clave}

La palabra "relaciones públicas" con sus variantes "relaciones" y "públicos" tiene presencia titular en el 36,7\% de los trabajos presentados. Le siguen, con un 29,5\%, los títulos con la palabra "comunicación". "Comunicación organizacional y/o empresarial", "comunicación corporativa" y "comunicación institucional" aparecen residualmente con un 3,1\%, 3\% y $2,2 \%$. Sin embargo, un porcentaje muy significativo de títulos $(25,5 \%)$ no incluyen ninguna de las palabras (unidades de registro). El análisis posterior de las palabras clave de los textos muestra que el 49,3\% incluye las palabras "públicos o relaciones" y el 50,7\%, la palabra "comunicación".

Los resultados concentran el término "relaciones públicas", "relaciones" o "públicos" en el congreso de la AIRP y, la palabra "comunicación", segundo registro más utilizado, alcanza mayor presencia en los trabajos del congreso de la $A E-I C$. La relación entre el título y el formato se calibra con el cálculo del coeficiente de contingencia $(C=0,460$ con una significación aproximada de $0<0,05)$. Se rechaza la HO de independencia y se concluye que ambas variables están relacionadas. El gráfico 1 recoge la presencia y la distribución de las palabras registradas en los títulos.

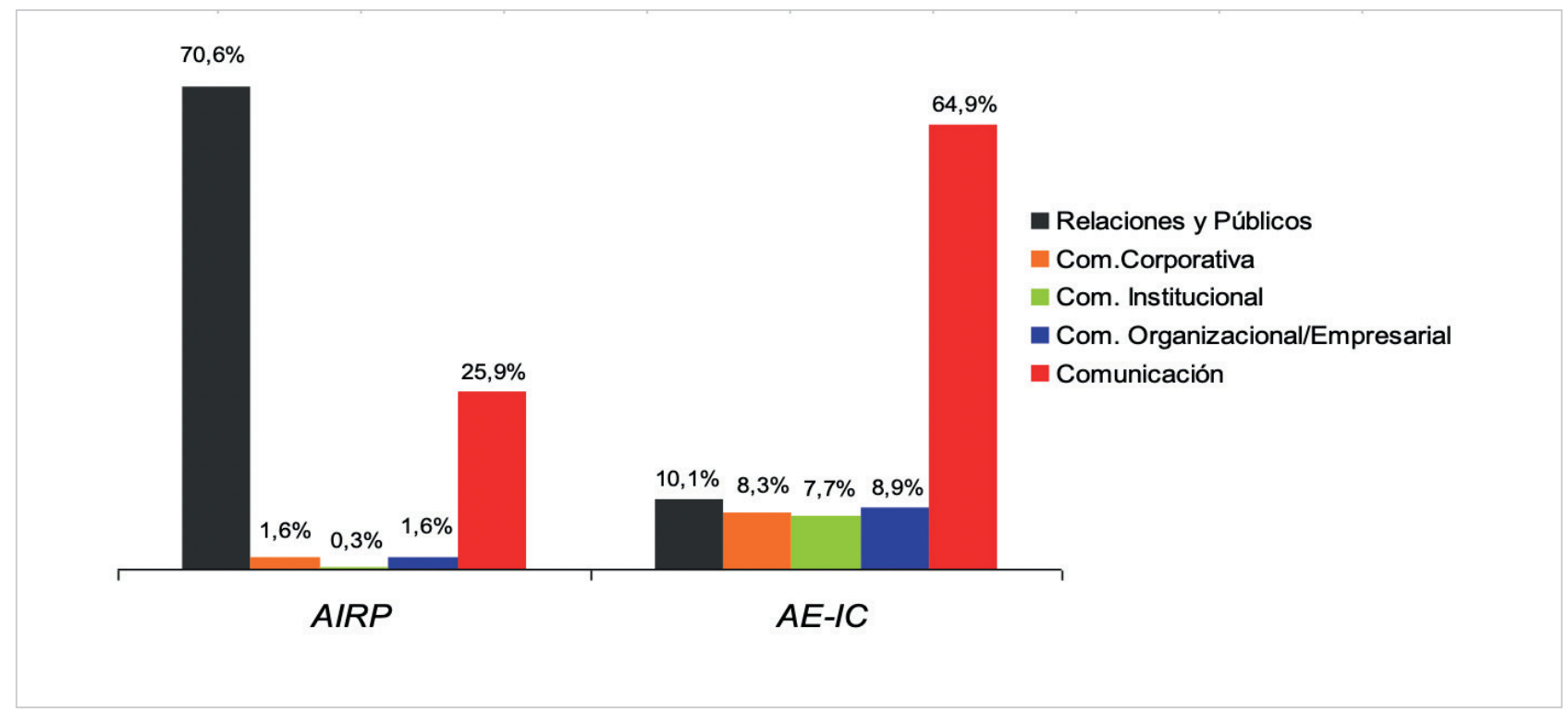

Gráfico 1. Palabras registradas en los títulos 


\subsubsection{Perfil y procedencia de autoría}

En total se han registrado 1.083 autores representados por mujeres en un $59,7 \%$ frente a los hombres con un $40,3 \%$. Sin embargo, esta distancia aumenta en casi $2 \%$ cuando las primeras firmantes son las mujeres $(61,1 \%$ de mujeres frente a un $38,9 \%$ de hombres). En el segundo orden de firmas, las mujeres aparecen en el $58,13 \%$ y los hombres en el $41,87 \%$. Los trabajos firmados por tres autores evidencian igualmente una mayoría de mujeres $(56,76 \%)$ mientras que los hombres aparecen en un $43,24 \%$.

Con respecto al país de procedencia de los firmantes, el $88 \%$ son autores españoles. El segundo país más representado, aunque a una gran distancia, es Brasil $(3,8 \%)$ y le siguen México $(2,7 \%)$ y Portugal $(1,7 \%)$. Costa Rica, en quinto lugar, tan sólo se encuentra en el $0,9 \%$ de los trabajos y Colombia en el $0,6 \%$. Comparten el séptimo puesto Estados Unidos, Argentina y Venezuela $(0,4 \%)$.

La autoría se clasifica en 3 categorías (universidades, entidad pública y empresa privada) donde resulta un total de 132 universidades.

Las universidades más prolíferas, atendiendo a la procedencia de los autores pueden verse en la tabla 1.

Considerando que la AIRP nació en el año 2004 con congresos anuales y que la $A E-I C$ en 2006 y sus congresos tienen una periodicidad bianual, la producción sobre relaciones públicas alcanza sus cuotas más altas en el 2010 y en el 2016 (gráfico 2). Es de señalar que la UMA y la UVIGO son dos universidades que destacan por concentrar trabajos, además, en los años 2011 y 2012 con respecto al resto de universidades del estudio.
Tabla 1. Universidades y número de trabajos producidos, según la afiliación de los autores

\begin{tabular}{|c|c|c|}
\hline & Universidad & Trabajos \\
\hline UMA & Universidad de Málaga & 114 \\
\hline UVIGO & Universidade de Vigo & 90 \\
\hline URV & Universitat Rovira i Virgili & 65 \\
\hline URLL & Universitat Ramon Llull & 59 \\
\hline UCM & Universidad Complutense de Madrid & 53 \\
\hline$U A$ & Universidad de Alicante & 48 \\
\hline US & Universidad de Sevilla & 46 \\
\hline URJC & Universidad Rey Juan Carlos & 38 \\
\hline$U A B$ & Universitat Autònoma de Barcelona & 33 \\
\hline UCAM & Univ. Católica San Antonio de Murcia & 27 \\
\hline UOC & Universitat Oberta de Catalunya & 29 \\
\hline USJ & Universidad San Jorge & 23 \\
\hline CEU & Universidad Ceu San Pablo & 22 \\
\hline UPF & Universitat Pompeu Fabra & 22 \\
\hline UVIC & Universitat de Vic-Central de Catalunya & 20 \\
\hline USC & Universidade de Santiago de Compostela & 19 \\
\hline$U C H-C E U$ & Universidad Cardenal Herrera, CEU & 18 \\
\hline UPV-EHU & Universidad del País Vasco & 16 \\
\hline UDC & Universidade de A Coruña & 16 \\
\hline IJ & Universitat Jaume I & 15 \\
\hline UNAV & Universidad de Navarra & 14 \\
\hline UNEX & Universidad de Extremadura & 13 \\
\hline UC3M & Universidad Carlos III & 10 \\
\hline UM & Universidad de Murcia & 10 \\
\hline$U B$ & Universidad de Barcelona & 9 \\
\hline$U C A$ & Universidad de Cádiz & 9 \\
\hline UDG-MX & Universidad de Guadalajara, México & 9 \\
\hline
\end{tabular}

La participación de autores en las contribuciones a congresos muestra un cambio sustancial en la línea temporal analizada (gráfico 3). Durante los primeros años, el número de autores en los trabajos era de 1 (línea azul). En 2009, descienden los textos de autoría única en beneficio de los de 2 y 3 autores. Además, a medida que avanza el análisis encontramos una tendencia al trabajo colaborativo. A partir del año 2013, los trabajos de 2 autores (línea roja) superan a los trabajos con un único autor y, a pesar de que las contribuciones con tres autores (línea verde) aún tiene poca presencia, en 2013 se vislumbra un cambio significativo que muestra una tendencia a la baja de trabajos de un solo autor. Desde 2014 y hasta 2016, las contribuciones de 2 autores igualan a las de 1 pudiendo considerarse el año 2017 como el

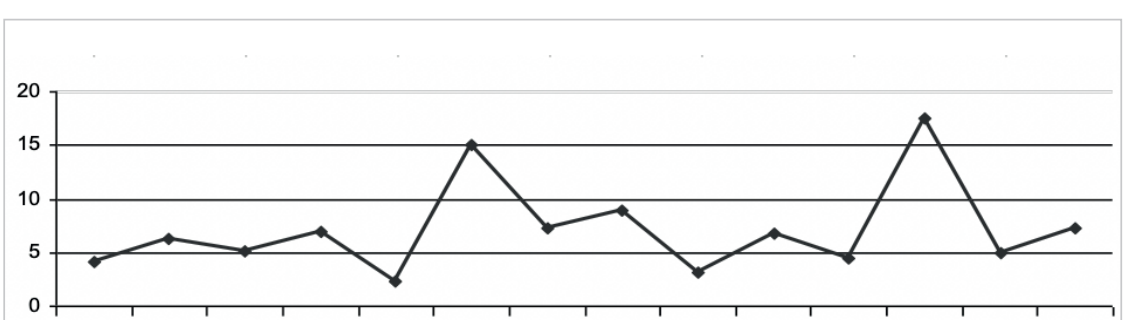

20042005200720082009201020112012201320142015201620172018

Gráfico 2. Distribución por año de las contribuciones sobre relaciones públicas

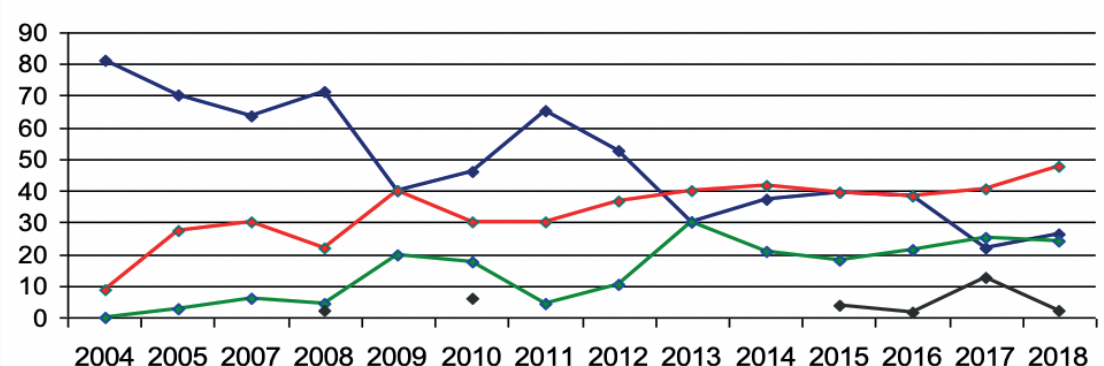

Gráfico 3. Evolución del número de autores por contribución: 1 autor, 2 autores, 3 autores. 
punto de inflexión en este aspecto, ya que predominan los trabajos de 2 autores, aumentan los de 3 autores y descienden los de 1 autor.

Para determinar la asociación entre año y número de autores, el estadístico chi-cuadrado revela que el $80 \%$ de los valores no es mayor que 5, por lo que se calcula el coeficiente de contingencia ( $C=0,371$, con una significación aproximada de 0 $<0,05)$ mostrando cierta relación aunque no muy intensa, y la prueba exacta de Fisher $(0,0)$ que es menor a los niveles normales, por lo que se rechaza la hipótesis nula $(\mathrm{HO})$ y se concluye que el año y número de autores están relacionadas.

Asimismo se evidencia un salto en la línea temporal con respecto al género de los primeros firmantes. Si bien desde 2004 y hasta 2010 la autoría logra una presencia media de 6 mujeres/4 hombres (constante lineal que se repite en las categorías de segundo y tercer orden de firma), entre 2011 y 2018, 7 de cada 10 primeros firmantes son mujeres.

\subsubsection{Temática y metodología}

Con respecto a la temática principal del estudio, cabe destacar que los autores tienden a escribir por orden sobre: relaciones institucionales y lobby $(12,8 \%)$; relaciones públicas y comunicación digital (12,3\%); relaciones públicas y estrategia $(11,3 \%)$; relaciones públicas y profesión $(8,6 \%)$; relaciones públicas y responsabilidad social (8,3\%); relaciones públicas y política (8\%); relaciones públicas y medios $(6,7 \%)$.

La temática secundaria, sin embargo, logra esta distribución: relaciones públicas y medios $(9,1 \%)$; relaciones públicas y comunicación digital (8.9\%); relaciones públicas y estrategia (7,8\%); relaciones públicas y profesión $(4,5 \%)$; relaciones públicas y teoría $(3,6 \%)$.

En su conjunto, las temáticas con mayor peso en los trabajos y que advierten del mayor interés investigador son: relaciones públicas y comunicación digital, estrategia de las relaciones públicas, relaciones con los medios de comunicación, relaciones institucionales y lobby y profesión de las relaciones públicas (tabla 2).

Descartando 148 trabajos no encontrados en los repositorios, el análisis del tipo de metodología utilizada se realiza sobre 492 textos. Los datos resuelven que el $84,76 \%$ cuenta con metodología descrita. De hecho, la aplicación mixta de métodos cualitativos y cuantitativos predomina en la investigación de relaciones públicas (gráfico 4).

El análisis entre la metodología y la temática de la investigación, partiendo de la hipótesis nula (HO) de que la temática no influye en el tipo de metodología utilizada, se calcula con la prueba exacta del test de Fisher $(0,0<0,05)$ concluyendo que la temática sí influye en el tipo de metodología utilizada en los trabajos de investigación y, por tanto, rechazamos la HO.

Los métodos utilizados (gráfico 5), como instrumentos de recogida de información para el desarrollo de las investigaciones, señalan al análisis

Tabla 2. Porcentajes de influjo de las temáticas de estudio en relaciones públicas. División en cuartiles

\begin{tabular}{|c|c|c|}
\hline \multicolumn{2}{|r|}{ Q1 } & $\%$ \\
\hline 1 & RPy comunicación digital & 14,7 \\
\hline 2 & RP Estrategia & 13,1 \\
\hline 3 & RP y medios & 10,9 \\
\hline 4 & RP Institucionales y lobby & 10,2 \\
\hline 5 & RP Profesión & 9,1 \\
\hline \multicolumn{2}{|r|}{ Q2 } & $\%$ \\
\hline 6 & RP y política & 6,5 \\
\hline 7 & RP y responsabilidad social & 6,3 \\
\hline 8 & RP Investigación & 5,3 \\
\hline 9 & RP Teoría & 5,2 \\
\hline 10 & RP y crisis & 4,2 \\
\hline \multicolumn{2}{|r|}{ Q3 } & $\%$ \\
\hline 11 & $\begin{array}{l}\text { RP Grados/estudios superiores en Publicidad y Rela- } \\
\text { ciones Públicas }\end{array}$ & 3,3 \\
\hline 12 & RP Internas & 3,2 \\
\hline 13 & RP y ceremonial & 2,9 \\
\hline 14 & RP y eventos & 2,6 \\
\hline 15 & RP y activismo & 1,3 \\
\hline \multicolumn{2}{|r|}{ Q4 } & $\%$ \\
\hline 16 & RP Internacionales & 0,5 \\
\hline 17 & RP Posgrados en comunicación y relaciones públicas & 0,3 \\
\hline 18 & RP Historia & 0,2 \\
\hline 19 & RP Financieras & 0,2 \\
\hline
\end{tabular}

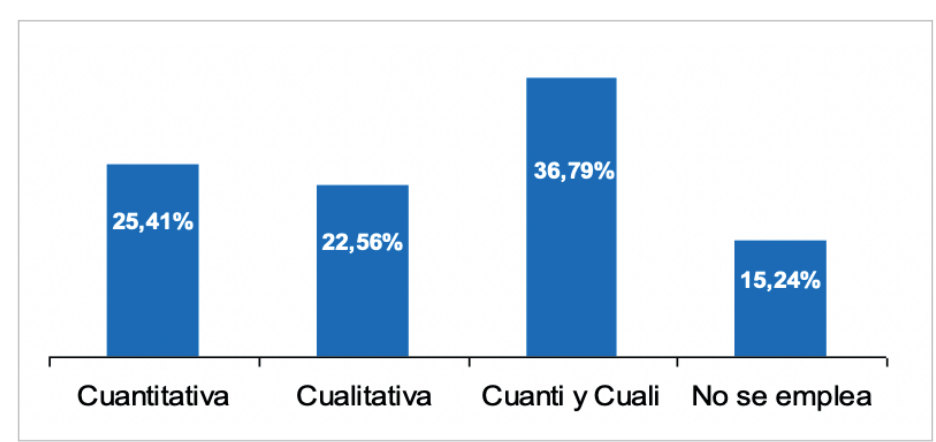

Gráfico 4. Metodología utilizada de contenido como la técnica de investigación más empleada por los investigadores. Es de destacar que el análisis de contenido se utiliza como método de investigación cuantitativo y cualitativo en el $55 \%$ de los casos; el $31,6 \%$ describe su aplicación cuantitativa y el 13,4\% lo aplica cualitativamente.

La entrevista en profundidad es el segundo método más utilizado y el cuestionario y la observación son utilizados en la misma proporción. El 22,3\% de los trabajos son estudios de caso. 


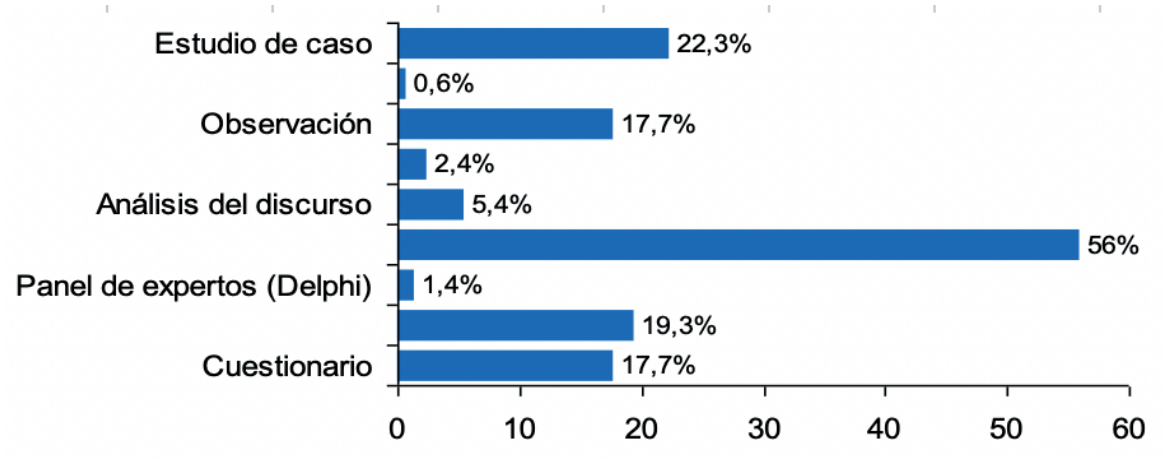

Gráfico 5. Métodos de investigación utilizados en las contribuciones a congresos

\subsection{Relaciones públicas en las tesis doctorales}

Se han analizado 103 tesis doctorales de relaciones públicas en el período comprendido entre 2005 y 2018 aprobadas en las universidades españolas.

Sobre la distribución entre universidades públicas y privadas, los datos muestran que la investigación en relaciones públicas recae por abrumadora mayoría en las universidad públicas ya que se han defendido y aprobado 86 tesis $(83,49 \%)$, frente a las 17 tesis aprobadas en universidades privadas (16,50\%).

Las universidades donde se han defendido más tesis doctorales se listan en la tabla 3.

Por lo que respecta al género de los estudiantes encontramos que las mujeres con un $58,25 \%$ son mayoría frente a un $41,74 \%$ de hombres. Este dato permite trasladar la cifra de que las mujeres son mayoría como estudiantes de grado, y que también se ha transferido al doctorado y a la investigación.

En el período analizado se observa que existe un quinquenio de altísima producción de tesis aprobadas ya que en los años 2013 a 2017 se aglutina el 74,75\% del total de tesis doctorales defendidas en las universidades. Una posible causa de esta concentración es la modificación de la normativa reguladora del doctorado que obligaba a la lectura de las tesis en un período determinado. Así, el año con mayor número de lecturas de tesis doctorales ha sido 2016 con un 19,41\% de las tesis de relaciones públicas.

Respecto del enfoque destacan los análisis de ámbito empresarial (27,18\%), el turismo y la política $(10,67 \%)$, deportes y redes sociales $(8,73 \%)$ y la comunicación interna $(7,76 \%)$. Cabe señalar que en casi todos los sectores analizados se implica el papel de las redes sociales para la construcción de la marca, el uso de las diferentes redes, la capacidad de cada red para la gestión de los públicos o la medición y el impacto de las redes.

El 95,14\% de las tesis doctorales implica una investigación, mientras que las tesis teóricas suponen el 4,85\%.

Por lo que respecta a la metodología de las tesis doctorales predominan las investigaciones de tipo mixto que aplica técnicas cualitativas y cuantitativas con un $61,16 \%$, seguida de los análisis que recurren a técnicas cualitativas con un $29,12 \%$ y, finalmente, las investigaciones cuantitativas con un $9,70 \%$. En este sentido, las principales técnicas utilizadas en las investigaciones son el análisis de contenido y las entrevistas.

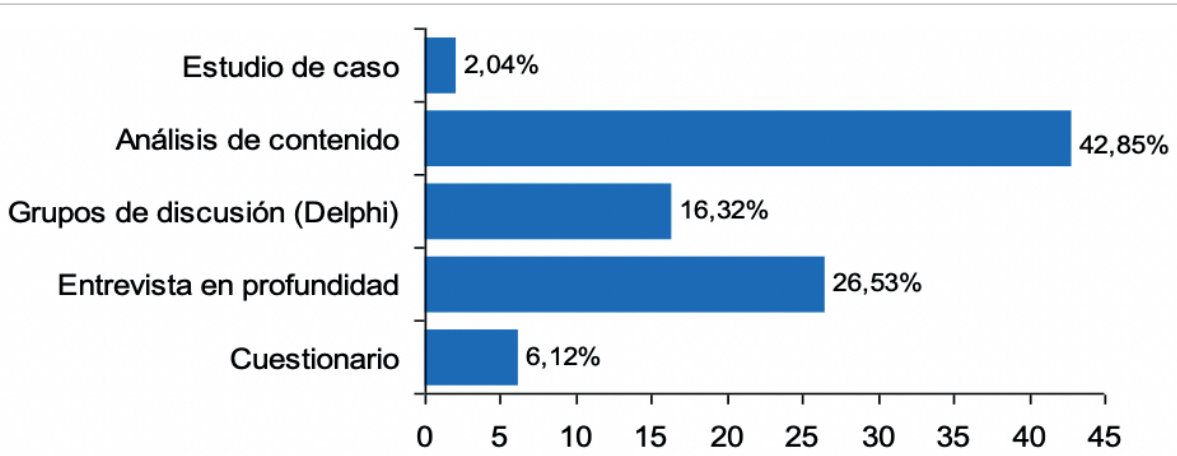

Gráfico 6. Métodos de investigación utilizados en las tesis doctorales 
En las tesis el principal método de investigación es el análisis de contenido con un 42,85\% de presencia. A este, por orden, le siguen las entrevistas, los grupos de discusión, los cuestionarios y los estudios de caso (gráfico 6).

\section{Discusión y conclusiones}

Con respecto al objetivo específico 1, analizar la presencia de la disciplina relaciones públicas, así como las temáticas objeto de estudio de los investigadores:

Los repositorios de la $A I R P$ y de la $A E-I C$ muestran un crecimiento ondeado en la investigación sobre relaciones públicas, alcanzando cuotas punta en los años 2010 y 2016. Las investigaciones cuyo título incluye relaciones públicas se concentran en los congresos de la AIRP y los trabajos de comunicación en general alcanzan mayor presencia en los congresos de la $A E-I C$, confirmando este último dato la tendencia a "invisibilizar" el término de relaciones públicas, especialmente en áreas genéricas de la comunicación (Míguez-González, 2011; Míguez-González; Baamonde-Silva; Corbacho-Valencia, 2014). Sin Los repositorios de la $A I R P$ y de la $A E-I C$ muestran un crecimiento ondeado en la investigación sobre relaciones públicas, alcanzando cuotas punta en los años 2010 y 2016

embargo, llama la atención que conceptos como comunicación corporativa, comunicación institucional y comunicación organizacional/empresarial no ganan tanta fuerza en los titulares en ninguno de los formatos (Molleda; Laskin, 2005; Castillo-Esparcia; Rubio-Moraga; Almansa-Martínez, 2012).

La presencia de las temáticas objeto de estudio en las contribuciones a los congresos y su exposición por cuartiles en un ranking (tabla 2) acumula más del 58\% (Q1) entre las cinco primeras de mayor interés y que son:

- relaciones públicas y comunicación digital,

- estrategia de las relaciones públicas,

- relaciones con los medios de comunicación,

- relaciones institucionales y lobby, y

- profesión de las relaciones públicas.

El siguiente grupo de temáticas (Q2) acopia el 27,5\% de los trabajos con una importante distancia de las del primer cuartil. Las temáticas del Q3 suman el 13,3\% y las temáticas del Q4 superan un justo 1,2\%.

Esta distribución constata que existe un elevado interés investigador por las temáticas del Q1, un interés bueno por las temáticas del Q2, interés bajo por las temáticas del Q3 y un interés residual por las temáticas del Q4. Todo ello en consonancia con estudios internacionales (Duhé, 2015; Wang, 2015).

En el caso de las tesis doctorales, si bien es cierto que el entorno profesional logra una gran significación, coincidiendo con la 5 a posición que ocupa en el Q1 del ranking de los congresos, la política consigue una representación superior. No obstante, la comunicación digital como objeto de análisis adquiere igualmente una presencia mayoritaria en las tesis lo que implica la constatación de su papel en las estrategias de relaciones públicas.

La consecución del objetivo específico 2, representar la tipología de los investigadores en relaciones públicas:

Aunque el carácter de los congresos es internacional, ambas asociaciones son españolas y es España donde se celebran desde sus inicios con la excepción del congreso de la AIRP de 2018 que se celebró en Portugal. Parece lógico que casi el $90 \%$ de los 1.083 académicos que presentan contribuciones en los congresos sean españoles y que el país de procedencia predominante sea España con exigua participación de investigadores de otros países.

Con respecto a la autoría, la firma de un único autor parece habitual en los inicios de las investigaciones. Sin embargo, en 2013 los trabajos compartidos por dos y tres firmantes comienzan a relegar a los trabajos de un autor (gráfico 3). La relación media entre hombres y mujeres es de 4 a 6 . Incluso, en la categoría de primer orden de firma, la proporción de 3 a 7 se mantiene durante la segunda mitad del período analizado.

La misma proporción se obtiene con los nuevos doctores, por cada 6 mujeres, hay 4 hombres. Es la constatación de la feminización de las relaciones públicas en la investigación.

La participación de los autores descubre a las Universidades de Málaga, Vigo, Rovira i Virgili, Ramon Llull, Complutense, Alicante, Sevilla, Rey Juan Carlos, Autònoma de Barcelona y Católica San Antonio de Murcia como las 10 universidades que más destacan en el objeto de estudio. Aunque el orden varía, las universidades con mayor volumen de tesis defendidas de relaciones públicas se encuentran entre las 10 primeras universidades con mayores contribuciones a congresos, con la Universidad de Málaga en primera posición, en la línea con estudios anteriores (Cuenca-Fontbona; Matilla; Compte-Pujol, 2019; Miquel-Segarra; Gonçalves, 2019).

La representatividad de metodología de investigación en el análisis de los datos indica un alto nivel de rigurosidad científica en las contribuciones sobre relaciones públicas en los congresos científicos y en las tesis doctorales 
Además, las contribuciones sobre relaciones públicas presentan una distribución más o menos constante a lo largo del período analizado excepto en 2010 y 2016 que, de manera significativa, van precedidos de un marcado descenso de producción (2009 y 2015).

En cuanto al objetivo específico 3, mostrar el grado de empirismo científico de las aportaciones, así como describir la metodología de investigación utilizada en materia de relaciones públicas:

La representatividad de metodología de investigación en el análisis de los datos indica un alto nivel de rigurosidad científica en las contribuciones sobre relaciones públicas en los congresos científicos y en las tesis doctorales. La combinación de metodologías cuantitativas y cualitativas describe de manera mayoritaria la investigación en este campo donde el análisis de contenido es el método más utilizado, seguido de la entrevista en profundidad en los congresos y en las tesis doctorales. El cuestionario, método eminentemente cuantitativo, comparte frecuencia de uso con la técnica de la observación en el caso de los congresos, pero queda en cuarto lugar de empleabilidad en las tesis doctorales en las que son más utilizados los grupos de discusión.

Con respecto al objetivo específico 4, averiguar las posibles relaciones entre las variables que analizan las tendencias investigadoras:

Los diferentes análisis y la relación calibrada entre algunas de las variables, evidencia una dependencia entre la presencia del término relaciones públicas y el formato, el año de publicación y el número de autores, y la metodología y la temática de las contribuciones.

Para concluir, el logro de los objetivos planteados permite emitir un diagnóstico en materia de relaciones públicas que se caracteriza por:

1. Investigaciones rigurosas que emplean metodologías mixtas con técnicas de la investigación de las ciencias sociales y en las que el análisis de contenido, como método principal, se aplica cuantitativa y cualitativamente.

2. Los investigadores más prolíferos se encuentran en las universidades donde el peso curricular de las relaciones públicas no es somero.

3. Las relaciones públicas son investigadas más por mujeres que por hombres, siendo ellas las primeras firmantes cuando la contribución es en colaboración con otros autores.

4. Los intereses investigadores se centran en las relaciones públicas y la comunicación digital, la estrategia de relaciones públicas, las relaciones con los medios, las relaciones institucionales y el lobby y la profesión de las relaciones públicas.

El hecho de que el presente estudio se centre en obtener una visión general de la investigación de las relaciones públicas en España a través del estudio de las tesis doctorales defendidas en el país (Teseo) y de las comunicaciones presentadas en los congresos de las dos principales asociaciones españolas en relaciones públicas y comunicación ( $A I R P$ y $A E-I C$ ), se debe a que se trata de la fuente y los foros más importantes del debate científico de la disciplina en España y referentes entre los miembros de la academia. El análisis de la producción científica en otros congresos de comunicación podría resultar interesante para tener una visión más amplia, aunque consideramos que los resultados obtenidos son suficientemente representativos y contribuyen al conocimiento de la investigación en relaciones públicas.

Tras lo expuesto, se ha logrado el objetivo principal de determinar, describir y analizar la investigación en relaciones públicas como objeto del debate científico de la disciplina en España.

\section{Referencias}

Andréu-Abela, Jaime (2000). Las técnicas de análisis de contenido: una revisión actualizada. Sevilla: Fundación Centro Estudios Andaluces.

Arceo-Vacas, José-Luis (2006). “La investigación de relaciones públicas en España”. Anàlisi, n. 34, pp. 111-124. https://core.ac.uk/download/pdf/38977760.pdf

Armendáriz, Enrique (2015). “El nuevo perfil del profesional de la Comunicación y las relaciones públicas. Una visión desde la perspectiva del mercado". Revista internacional de relaciones públicas, v. 5, n. 9, pp. 153-178.

https://doi.org/10.5783/RIRP-9-2015-09-153-178

Castillo-Esparcia, Antonio (2011). "El rol de las publicaciones científicas en Comunicación en el EEES: indexación e impacto". Revista internacional de relaciones públicas, v. 1, n. 1, pp. 135-154.

https://doi.org/10.5783/RIRP-1-2011-07-135-154

Castillo-Esparcia, Antonio; Carretón-Ballester, Carmen (2010). “Investigación en comunicación. Estudio bibliométrico de las revistas de comunicación en España”. Comunicación y sociedad, v. 23, n. 2, pp. 289-327.

http://hdl.handle.net/10171/27872 
Castillo-Esparcia, Antonio; Rubio-Moraga, Ángel; Almansa-Martínez, Ana (2012). "La investigación en Comunicación. Análisis bibliométrico de las revistas de mayor impacto del ISI". Revista latina de comunicación social, n. 67, pp. 248-270. https://doi.org/10.4185/RLCS-067-955-248-270

Castillo-Esparcia, Antonio; Xifra, Jordi (2006). "Investigación bibliométrica de las tesis doctorales españolas sobre relaciones públicas". Anàlisi, n. 34, pp. 141-161.

https://ddd.uab.cat/record/15568

Compte-Pujol, Marc; Matilla, Kathy; Hernández, Salvador (2018). “Estrategia y relaciones públicas: un estudio bibliométrico comparativo". Revista latina de comunicación social, n. 73, pp. 748-764.

https://doi.org/10.4185/RLCS-2018-1280

Cuenca-Fontbona, Joan; Matilla, Kathy; Compte-Pujol, Marc (2019). “Análisis bibliométrico de la producción científca de las relaciones públicas a través de las tesis doctorales (2006-2017) y de los programas de doctorado españoles (2017)". Anàlisi, n. 60, pp. 97-117.

https://doi.org/10.5565/rev/analisi.3166

Davidson, Scott (2015). "Everywhere and nowhere: Theorising and researching public affairs and lobbying within public relations scholarship". Public relations review, v. 41, n. 5, pp. 615-627.

https://doi.org/10.1016/j.pubrev.2014.02.023

Dircom (2018). El estado de la comunicación en España. Madrid: Dircom.

http://www.dircom.org/actualidad-dircom/item/9176-estado-de-la-comunicacion-en-espana-2018

Duhé, Sandra (2012). "A thematic analysis of thirty years of public relations literature addressing the potential and pitfalls of new media". In: Duhé, Sandra (ed.). New media and public relations, $2^{\text {nd }}$ edition. New York: Peter Lang, pp. 57-66. ISBN: 9781433101243

Duhé, Sandra (2015). "An overview of new media research in public relations journals from 1981 to 2014". Public relations review, v. 41, n. 2, pp. 153-169.

https://doi.org/10.1016/j.pubrev.2014.11.002

Fernández-Quijada, David (2011a). "Appraising internationality in Spanish communication journals”. Journal of scholarly publishing, v. 43, n. 1, pp. 90-109.

https://doi.org/10.3138/jsp.43.1.90

Fernández-Quijada, David (2011b). “De los investigadores a las redes. Una aproximación tipológica a la autoría en las revistas españolas de comunicación". En: $1^{\text {er }}$ Congreso nacional de metodología de la investigación en comunicación, pp. 1-15. ISBN: 9878469427132

http://eprints.rclis.org/18393/1/2011_aeic.pdf

Ha, Jin-Hong; Riffe, Daniel (2015). "Crisis-related research in communication and business journals: An interdisciplinary review from 1992 to 2011". Public relations review, v. 41, n. 4, pp. 569-578.

https://doi.org/10.1016/j.pubrev.2015.06.019

Hellín-Ortuño, Pedro-Antonio; García-López, Javier (2011). "La integración de la publicidad y las relaciones públicas en el Espacio Europeo de Educación Superior". En: Fernández, Ana-Belén; García, Francisco (eds.). VI Congreso internacional de investigación y relaciones públicas, pp. 96-106. ISBN: 9788493907716

http://airrpp.org/wp-content/uploads/2012/12/actas_VIcongreso_AIRP.pdf

Hernández, Salvador; Losada, José-Carlos; Matilla, Kathy (2009). "Las relaciones públicas y la comunicación corporativa en la oferta universitaria de postgrado del estado español: análisis sistemático y proyectivo y una iniciativa de observatorio del estado de la situación por parte de una asociación profesional". Razón y palabra, v. 14, n. 70, pp. 1-18. https://www.redalyc.org/pdf/1995/199520478006.pdf

Huertas, Assumpció; Fernández-Cavia, José (2006). “Center and periphery: two speeds for the implementation of Public Relations in Spain". Public relations review, v. 32, n. 2, pp. 110-117.

https://doi.org/10.1016/j.pubrev.2006.02.003

Ki, Eyun-Jung; Shin, Jae-Hwa (2006). "Status of organization - public relationship research from an analysis of published articles, 1985-2004". Public relations review, v. 32, n. 2, pp. 194-195.

https://doi.org/10.1016/j.pubrev.2006.02.019

Kim, Jeong-Nam; Ni, Lan (2013). "Two types of Public Relations problems and integrating formative and evaluative research: A review of research programs within the behavioral, strategic management paradigm". Journal of public relations research, v. 25, n. 1, pp. 1-29.

https://doi.org/10.1080/1062726X.2012.723276 
Kim, Sora; Avery, Elizabeth J.; Lariscy, Ruthann W. (2009). "Are crisis communicators practicing what we preach?: An evaluation of crisis response strategy analyzed in public relations research from 1991 to 2009 ". Public relations review, v. 35, n. 4, pp. $446-448$. https://doi.org/10.1016/j.pubrev.2009.08.002

Liu, Brooke-Fisher; Bartz, Logen; Duke, Noreen (2016). "Communicating crisis uncertainty: A review of the knowledge gaps". Public relations review, v. 42, n. 3, pp. 479-487.

https://doi.org/10.1016/j.pubrev.2016.03.003

Martínez-Nicolás, Manuel; Saperas-Lapiedra, Enric (2011). “La investigación sobre Comunicación en España (1998-2007). Análisis de los artículos publicados en revistas científicas”. Revista latina de comunicación social, n. 66, pp. 111-129. https://doi.org/10.4185/RLCS-66-2011-926-101-129

Martínez-Nicolás, Manuel; Saperas-Lapiedra, Enric (2016). “Objetos de estudio y orientación metodológica de la reciente investigación sobre comunicación en España (2008-2014): Análisis de los trabajos publicados en revistas científicas españolas". Revista latina de comunicación social, n. 71, pp. 1365-1384.

https://doi.org/10.4185/RLCS-2016-1150

Martínez-Nicolás, Manuel; Saperas-Lapiedra, Enric; Carrasco-Campos, Ángel (2019). "La investigación sobre comunicación en España en los últimos 25 años (1990-2014). Objetos de estudio y métodos aplicados en los trabajos publicados en revistas españolas especializadas". Empiria. Revista de metodología de ciencias sociales, n. 42, pp. 37-69.

https://doi.org/10.5944/empiria.42.2019.23250

Masip, Pere (2011). "Efecto Aneca: producción española en comunicación en el Social Science Citation Index". Anuario ThinkEPI, v. 5, pp. 206-210.

https://recyt.fecyt.es/index.php/ThinkEPI/article/view/30505/16061

Matilla, Kathy; Cuenca-Fontbona, Joan; Compte-Pujol, Marc (2018). “Un análisis de las asignaturas sobre Comunicación y relaciones públicas en los masters universitarios españoles tipo MBA (curso 2016-2017)”. adComunica, n. 16, pp. 221-240. https://doi.org/10.6035/2174-0992.2018.16.12

Matilla, Kathy; Hernández, Salvador (2011). “Bolonia primer año: los estudios universitarios de RRPP en Cataluña en el curso 2010-11". En: Fernández, Ana-Belén; García, Francisco (eds.). VI Congreso internacional de investigación y relaciones públicas, pp. 35-59. ISBN: 9788493907716

http://airrpp.org/wp-content/uploads/2012/12/actas_VIcongreso_AIRP.pdf

Matilla, Kathy; Hernández, Salvador (2012). “Bolonia 2o año: los estudios universitarios de relaciones públicas en Cataluña en el curso 2011-2012". Revista internacional de relaciones públicas, v. 2, n. 4, pp. 247-276. http://revistarelacionespublicas.uma.es/index.php/revrrpp/article/view/117

Matilla, Kathy; Hernández, Salvador; Compte-Pujol, Marc (2018). “Modelos profesionales y grados universitarios de Publicidad y relaciones públicas en España desde la perspectiva de las relaciones públicas (2017-2018)". Communication papers, v. 7, n. 15, pp. 91-104.

https://doi.org/10.33115/udg_bib/cp.v7i15.22185

Matilla, Kathy; Hernández, Salvador; Losada, José-Carlos (2010). “La oferta formativa de relaciones públicas en los nuevos grados universitarios en España". Pangea, n. 1, pp. 124-159.

https://revistaraic.files.wordpress.com/2010/12/01-01-107.pdf

McAllister-Spooner, Sheila M. (2009). "Fulfilling the dialogic promise: A ten-year reflective survey on dialogic internet principles". Public relations review, v. 35, n. 3, pp. 320-322.

https://doi.org/10.1016/j.pubrev.2009.03.008

McCorkindale, Tina; DiStaso, Marcia W. (2014). "The state of social media research: where we are now, where we were and what it means for public relations". Research journal of the Institute for Public Relations, v. 1, n. 1, pp. 1-17. https://instituteforpr.org/wp-content/uploads/TinaMarciaWES.pdf

Meadows, Cui; Meadows, Charles-Williams (2014). "The history of academic research in public relations: Tracking research trends over nearly four decades". Public relations review, v. 40, n. 5, pp. 871-873.

https://doi.org/10.1016/j.pubrev.2014.06.005

Míguez-González, María-Isabel (2011). “La investigación sobre relaciones públicas en las revistas españolas de comunicación”. En: Fernández, Ana-Belén; García, Francisco (eds.). VI Congreso internacional de investigación y relaciones públicas, pp. 134-138. ISBN: 9788493907716

http://airrpp.org/wp-content/uploads/2012/12/actas_VIcongreso_AIRP.pdf

Míguez-González, María-Isabel; Baamonde-Silva, Xosé-Manuel; Corbacho-Valencia, Juan-Manuel (2014). “A bibliographic study of public relations in Spanish media and communication journals, 2000-2012". Public relations review, v. 40, n. 5, pp. 818-828.

https://doi.org/10.1016/j.pubrev.2014.08.002 
Míguez-González, María-Isabel; Corbacho-Valencia, Juan-Manuel; Baamonde-Silva, Xosé-Manuel (2016). “Tendencias de investigación sobre relaciones públicas en revistas internacionales: el caso del 'Journal of public relations research' 2012-2014". Revista internacional de relaciones públicas, v. 6, n. 12, pp. 5-24.

https://doi.org/10.5783/RIRP-12-2016-02-05-24

Míguez-González, María-Isabel; Costa-Sánchez, Carmen (2019). “Tendencias de investigación sobre comunicación organizacional: la autoría española en revistas indexadas (2014-2018)". El profesional de la información, v. 28, n. 5, pp. $12-27$. https://doi.org/10.3145/epi.2019.sep.10

Miquel-Segarra, Susana; Gonçalves, Gisela (2019). “Análisis bibliométrico de tesis doctorales sobre relaciones públicas en España y Portugal (2006/2016)". Revista internacional de relaciones públicas, v. 9, n. 17, pp. 25-48.

https://doi.org/10.5783/RIRP-17-2019-03-25-48

Molleda, Juan-Carlos; Laskin, Alexander V. (2005). Global, international, comparative and regional public relations knowledge from 1990 to 2005: A quantitative content analysis of academic and trade publications. The Institute for Public Relations.

https://instituteforpr.org/wp-content/uploads/Int_PR_Knowledge.pdf

Morehouse, Jordan; Saffer, Adam-John (2018). "A bibliometric analysis of dialogue and digital dialogic research: mapping the knowledge construction and invisible colleges in public relations research". Journal of public relations research, v. 30, n. 3, pp. 65-82. https://doi.org/10.1080/1062726X.2018.1498343

Moreno-Fernández, Ángeles; Carrasco-Campos, Ángel; Saperas-Lapiedra, Enric (2014). "Los efectos del proceso Bolonia en el currículum de las relaciones públicas. Análisis del estado de la cuestión”. Sphera publica, n. junio, pp. 163-184. http://sphera.ucam.edu/index.php/sphera-01/article/view/207

Morkunas, Vida J.; Moore, Shane; Duncan, Sherese Y. (2018). "A bibliometric analysis of the Journal of public affairs". Journal of public affairs, v. 19, n. 1, pp. 1-17.

https://doi.org/10.1002/pa.1833

Noguero-Grau, Antoni (2004). "Principales acontecimientos en la evolución de las relaciones públicas en España”. En: Arceo-Vacas, José-Luis (dir.). Las relaciones públicas en España. Madrid: McGraw-Hill, pp. 337-359. ISBN: 8448141830

Perlado-Lamo-de-Espinosa, Marta; Saavedra-Llamas, Marta (eds.) (2017). Los estudios universitarios especializados en Comunicación en España. Un análisis desde la perspectiva profesional de la Dirección de Comunicación (2014-2015). Barcelona: UOC, Colección Dircom. ISBN: 9788491167013

Santa-Soriano, Alba; Lorenzo-Álvarez, Carolina; Torres-Valdés, Rosa-María (2018). "Bibliometric analysis to identify an emerging research area: Public Relations Intelligence - a challenge to strengthen technological observatories in the network society". Scientometrics, v. 115, n. 3, pp. 1591-1614.

https://doi.org/10.1007/s11192-018-2651-8

Sisco, Hilary F.; Pressgrove, Geah; Collins, Erik L. (2013). "Paralleling the practice: an analysis of the scholarly literature in nonprofit Public Relations". Journal of public relations research, v. 25, n. 4, pp. 282-306.

https://doi.org/10.1080/1062726X.2013.806869

Theunissen, Petra; Noordin, Wan-Norbani-Wan (2012). "Revisiting the concept 'dialogue' in public relations". Public relations review, v. 38, n. 1, pp. 5-13.

https://doi.org/10.1016/j.pubrev.2011.09.006

Vanc, Antoaneta M.; Fitzpatrick, Kathy R. (2016). "Scope and status of public diplomacy research by Public Relations scholars, 1990-2014". Public relations review, v. 42, n. 3, pp. 432-440.

https://doi.org/10.1016/j.pubrev.2015.07.012

Wang, Yuan (2015). “Incorporating social media in Public Relations: A synthesis of social media-related Public Relations research". Public relations journal, v. 9, n. 3, pp. 1-14.

https://prjournal.instituteforpr.org/wp-content/uploads/2015v09n03Yang.pdf

Xifra, Jordi (2007). "Undergraduate public relations education in Spain: Endangered species?". Public relations review, v. 33, n. 2, pp. 206-213.

https://doi.org/10.1016/j.pubrev.2007.02.006

Xifra, Jordi; Castillo-Esparcia, Antonio (2006). "Forty years of doctoral Public Relations research in Spain: A quantitative study of dissertation contribution to theory development". Public relations review, v. 32, n. 3, pp. 302-308.

https://doi.org/10.1016/j.pubrev.2006.05.004

Ye, Lan; Ki, Eyun-Jung (2012). "The status of online public relations research: An analysis of published articles in $1992-$ 2009". Journal of public relations research, v. 24, n. 5, pp. 409-434.

https://doi.org/10.1080/1062726X.2012.723277 